\section{A Obesidade Estaria Relacionada ao Aumento do Volume das Advenais?}

\begin{abstract}
RESUMO
Vários estudos sugerem que existe uma hiperatividade do eixo hipotálamo-hipofisário-adrenal (HHA) na obesidade, com maior acúmulo de gordura na região abdominal. Estes trabalhos demonstram que, após injeções de hormônio liberador de corticotropina (CRF) ou hormônio adrenocorticotrófico (ACTH) e ainda através de testes de stress, os níveis de cortisol estão aumentados em comparação com pacientes com deposição periférica de gordura. Além do mais, alguns estudos mostram que em pacientes deprimidos, onde a hiperatividade do HHA é uma alteração endócrina importante e conhecida, o volume das adrenais está aumentado quando comparado com normais. Para investigar se o teor de gordura visceral de alguma maneira se relaciona com o volume das adrenais, 52 mulheres com idade entre 19 e 54 anos, com diferentes indices de corpulência, foram estudadas através de medidas antropométricas como peso, índice de massa corporal (IMC), cintura e relação cintura-quadril (RCQ). As áreas de gordura visceral ( $(S V)$ e subcutânea (GSC), além do volume das adrenais, foram medidas através da tomografia computadorizada. Houve uma correlação extremamente significativa entre as medidas de distribuição de gordura ( $R C Q$ e cintura) e a GV, sendo que a RCQ não se correlacionou com a GSC. O somatório do volume das adrenais mostrou uma correlação positiva e significativa com a $R C Q(r=0,272, p=0,02)$ e uma correlação positiva, mas no limite da significância com a $G V(r=0,228, p=0,05)$, não mostrando qualquer correlação com a GSC. Além disso, o somatório do volume das adrenais foi maior naquelas com $\mathrm{GV} \geq 120 \mathrm{~cm}^{2}$ quando comparado com pacientes com área de $G V<120 \mathrm{~cm}^{2}(p=0,05)$. Portanto, o estudo sugere que o depósito de GV parece inter-relacionar-se com a hiperatividade do HHA, aqui estimada anatomicamente através do volume das adrenais, glândula alvo deste eixo. (Arq Bras Endocrinol Metab 2000;44/1: 21-29)
\end{abstract}

Unitermos: Obesidade; Adrenais; Eixo hipotálamo-hipófise-adrenal; Cortisol; Tomografia computadorizada

\begin{abstract}
Many studies suggest that there is a hyperactivity of the hypothalamicpituitary-adrenal (HPA) axis related to obesity with accumulation of fat in the abdominal region. Some studies demonstrate that after the injection of corticotrophin-releasing hormone (CRH) or corticotrophin (ACTH) and according to the results of stress tests, the levels of cortisol are increased when compared to patients with peripheral fat deposition. Moreover, some studies show that in depressed patients, where the hyperactivity of HPA is known and represents an important endocrinological change, the adrenal volume is increased. To investigate if the amount of visceral fat is related in some way to adrenal gland volume, a group of 52 women with different corpulence indexes was studied. Anthropometrics measures, such as weight, body mass index (BMI), waist circumference and waist-to-hip ratio (WHR) were checked. The areas of visceral and subcutaneous fat, as well as adrenal gland volumes, were checked by abdominal computed tomography. There was an extremely relevant correlation between the measurements of central fat deposition (WHR
\end{abstract}

\section{artigo original}

\author{
Amélio F.G. Matos \\ Andréa R. Vieira \\ Walmir Coutinho \\ Denise Madeira \\ Lúcia M. Carraro \\ Rosa Rodrigues \\ Gláucia Bastos \\ Mônica Cabral \\ André Pantaleão \\ Jucinéia Oliveiva \\ Ricardo M.R. Meivelles
}

Instituto Estadual de Diabetes e Endocrinologia Luiz Capriglione . Serviço de Radiologia do Dr. Amarino C. de Oliveira do Hospital Pró-Cardíaco. 
and waist circumference) and the amount of visceral fat (VF), although no correlation was found between the WHR and the subcutaneous fat. The added adrenals volumes showed a positive correlation to the WHR ( $r=0.272 ; p=0.02)$ and also to the VF $(r=0.228 ; p=0.05)$. No correlation with the subcutaneous fat, $\mathrm{BMl}$, and weight were found. The added adrenals volumes were increased when VF $\geq 120 \mathrm{~cm}^{2}$ as compared with patients with VF $<120 \mathrm{~cm}^{2}(\mathrm{p}=$ 0.05). Thus, this study suggests that the amount of VF seem to be related to the HPA hyperactivity, anatomically expressed in this study by the adrenal volume, the target gland of this axis. (Arq Bras Endocrinol Metab 2000;44/1: 21-29)

Keywords: Obesity; Adrenal glands; Hypothalamichypophyseal-adrenal axis; Cortisol; Computerized tomography

A DISTRIBUIÇ̃o DA GORDURA CORPORAL, especialmente a gordura abdominal ou o acúmulo de gordura na parte superior do corpo (obesidade central), tem sido mais intensamente associada à morbidade-mortalidade do que a obesidade da parte inferior do corpo ou o grau de obesidade (1).

A maior contribuição para esta conclusão foi o trabalho pioneiro de Jean Vague, realizado nos anos 40. Vague foi o primeiro a distinguir entre os dois tipos de obesidade de acordo com a distribuição da gordura, e a afirmar que a distribuição de gordura do tipo andróide, isto é, a que se localiza na parte superior do corpo (obesidade andróide), estava associada à maioria das complicações da obesidade, como diabetes mellitus e arteriosclerose e que o depósito na parte inferior (obesidade ginóide) observada, principalmente, em mulheres é "benigna" (2).

A importância da distribuição da gordura corporal, como um indicador de risco, foi confirmada pelas notáveis contribuiçōes de Björntörp (3) e Kissebah (4), que acarretou a inclusão da distribuição da gordura entre os fatores de risco estabelecidos de doença cardiovascular e de mortalidade. Estudos prospectivos que se seguiram, demonstraram que uma maior proporção de gordura na região abdominal estava associada a um risco aumentado de dislipidemia, diabetes e doença cardiovascular (4-12). Além disso, esta relação foi demonstrada como sendo parcialmente independente do conteúdo total de gordura. Para um mesmo IMC (índice de massa corporal), a obesidade abdominal está associada a um risco muito maior de desenvolvimento de doença concomitante do que a obesidade da parte inferior do corpo.

Do ponto de vista clínico, a estimativa da dis tribuição regional de gordura deve ser de grande importância no paciente obeso. Atualmente, a relação entre a circunferência da cintura/quadril (RCQ) é a medida antropométrica mais usada como índice de regionalização do tecido adiposo. Entretanto, não diferencia de maneira precisa o tecido subcutâneo do visceral. Assim sendo, as técnicas de imagem informam com precisão a quantidade de gordura intra-abdominal e subcutânea. Através da análise por tomografia computadorizada (TC), muitos estudos vêm demonstrando que a gordura localizada dentro da cavidade abdominal, gordura visceral (GV), é a mais crítica e correlacionada com os distúrbios metabólicos (13-18). A maioria dos estudos têm mostrado correlação importante entre a RCQ e a quantidade de GV mensurada por TC (19-22). A cintura é outra medida de distribuição de gordura e que, também, tem forte correlação com a GV (20,22-25). Contudo, o uso somente da circunferência da cintura pode apresentar um obstáculo importante. Para uma dada circunferência de cintura, alguns pacientes terão uma quantidade aumentada de gordura visceral, enquanto que outros o conteúdo maior pode ser subcutâneo.

Um grande número de alterações endócrinas estão presentes na obesidade e são mais pronunciadas na obesidade visceral que na periférica, glúteo-femural. As evidências sugerem que exista uma origem neuroendócrina central para tais alterações. A disfunção central, primária, na obesidade visceral parece ser a hipersensibilidade e/ou hiper-responsividade do eixo hipotálamo-hipofisário-adrenal (HHA), levando muitos estudos a esta conclusão. Demonstra-se que a concentração de cortisol livre urinário (CLU) está elevada na obesidade central (26). Atribui-se esta elevação, primeiramente, ao clearence aumentado do cortisol, não importando se a obesidade é abdominal ou periférica (27). A segunda razão seria a hipersensibilidade do HHA a fatores estressantes físicos e mentais (2830). Além disso foi observado uma diminuição da inibição do cortisol pela dexametasona, em obesos com clevada RCQ (31), novamente sugerindo uma hipersensibilidade do eixo HHA, devido a uma diminuída inibição por feed back via receptores de glicocorticóides ( $R G$ ) à nível de sistema nervoso central (SNC).

Todas estas conclusões, baseadas em resultados de detalhados estudos celulares e moleculares, são fortemente enfatizadas pelas várias observações clínicas. $\mathrm{O}$ desequilíbrio entre os hormônios acumuladores de lipídios, cortisol e insulina, e o grupo de hormônios mobilizadores de lipídios, são encontrados em várias condições de acúmulo de gordura visceral, como na síndrome de Cushing (cortisol e insulina elevados e baixas concentrações de hormônios sexuais e GH), na menopausa e no avanço da idade (baixas concen- 
trações de GH e de hormônios sexuais), no alcoolismo (periodicamente, níveis elevados de cortisol e baixos de hormônios sexuais), no tabagismo (periodicamente, elevadas concentrações de cortisol), assim como na ansiedade e depressão (elevados níveis de cortisol) (32). A correção destas anormalidades é seguida por diminuição ou mesmo normalização do conteúdo de gordura visceral. Portanto, estas evidências sugerem que as alterações endócrinas observadas na obesidade visceral, provavelmente, são causadoras do desproporcional acúmulo de gordura visceral (32).

Alguns estudos demonstram que nos estados de depressão maior, onde a hiperatividade do eixo HHA é a mais proeminente anormalidade neuroendócrina $(33,34)$, o volume das glândulas adrenais está aumentado, em aproximadamente $70 \%$, quando comparado com o volume destas, após o tratamento e, também, significativamente maior, quando comparado com voluntários normais (35). Este estudo é concordante com trabalho prévio, que demonstra uma hiperplasia adrenal em pacientes com depressão (36).

Apesar das claras evidências que ligam o eixo HHA à obesidade central c/ou visceral, e, também, apesar da demonstração do aumento do volume adrenal em pacientes com depressão, não existem na literatura estudos direcionados para a avaliação anatômica das adrenais na obesidade. Este estudo hipotetiza que, havendo uma hiperatividade do HHA, poderia haver na obesidade visceral alguma associação com o volume desta glândula. Este estudo visa, portanto, demonstrar, através da correlação da área de gordura intra-abdominal com o volume das adrenais, uma associação anatômica da disfunção deste eixo.

\section{MATERIAIS E MÉTODOS}

Quarenta e sete mulheres obesas ou com excesso de peso, foram recrutadas no ambulatório de obesidade do Instituto Estadual de Diabetes e Endocrinologia Luiz Caprigione. Cinco mulheres normais foram recrutadas entre as acompanhantes das pacientes obesas, totalizando 52 voluntárias. Todas concordaram $\mathrm{em}$ participar do estudo, assinando um documento de consentimento. A idade média foi de 37 anos, variando de 19 a 54 anos. Todas estavam livres de doenças concomitantes importantes como diabetes mellitus, doença coronariana, doenças hepáticas, renais, endócrinas, com especial atenção a sinais de hipercortisolismo e câncer. O estudo nas mulheres em fase pré-menopáusica foi realizado, sem levar em consideração a fase menstrual.

Todas as voluntárias estavam com peso estável (não houve ganho ou perda maior que $2 \mathrm{~kg}$ ) nos últi- mos 6 meses e neste período não fizeram uso de qualquer medicação com o objetivo de perder peso.

As voluntárias recrutadas foram avaliadas clinicamente c antropometricamente, com aferição do peso, altura, medida da cintura e do quadril. A cintura foi mensurada à nível do menor diâmetro, entre o rebordo costal e as cristas ilíacas, e o quadril ao nível do maior diâmetro, passando pelos grandes trocanteres. $O$ índice de massa corporal (IMC) foi calculado, através da fórmula peso $(\mathrm{kg}) /$ altura $(\mathrm{m})^{2}$ e a relação cintura-quadril $(\mathrm{RCQ})$, através da fórmula cintura $(\mathrm{cm}) /$ quadril $(\mathrm{cm})$.

Todas as mulheres foram submetidas a tomografia computadorizada abdominal para medir o volume das adrenais, a gordura visceral e subcutânea (37). Todo o estudo tomográfico foi realizado pela mesma radiologista, Dra. Denise Madeira, no Hospital Pró-Cardíaco, Serviço de Radiologia do Dr. Amarino C. de Oliveira.

$\mathrm{O}$ estudo tomográfico das supra-renais e do abdômen foi realizado em sistema helicoidal, sem contraste oral ou venoso, em equipamento modelo PROSPEED, da marca GE. O protocolo para o estudo das supra-renais inclui aquisição helicoidal, em apnéia única inspiratória, com cortes de $5 \mathrm{~mm}$ de espessura, $5 \mathrm{~mm}$ de espaçamento entre os cortes, $p i t c h=1, F O V=50 \mathrm{~cm}$. Para a avaliação das gorduras abdominal total, visceral e subcutânea foi realizada pequena hélice de 4 cortes, com $10 \mathrm{~mm}$ de espessura e $10 \mathrm{~mm}$ de espaçamento, mantendo o mesmo pitch e FOV ao nível de L4-L5.

Após incluído o grupo como um todo para a correlação estatística, o distribuímos entre pacientes com gordura visceral $<\mathrm{e} \geq 120 \mathrm{~cm}^{2}$.

Para análise estatística, foram estudadas as distribuições das diversas variáveis presentes no estudo a fim de averiguar o tipo de distribuição, bem como as medidas de tendência central das mesmas. A comparação entre as diversas variáveis ordinais foi realizada através da correlação parcial de Pearson, que variava de -l (correlação negativa ou inversa) a +1 (correlação positiva ou direta), testando-se, posteriormente, a significância estatística das duas variáveis correlacionadas, através da transformação $\mathrm{Z}$ de Fischer. A comparação das médias entre as duas categorias de gordura visceral foram realizadas através do teste $t$ de Student, desde que a igualdade das variâncias entre os grupos testados (teste de Levene) fosse comprovada. Em caso de diferirem, formas não-paramétricas deste teste foram realizadas (teste de Scheffé). O nível de significância foi de $\mathrm{p}<0,05$.

\section{RESULTADOS}

Na Tabela 1 estão demonstrados a idade e os dados antropométricos e na Tabela 2 os dados tomográficos, através das médias e seus desvios padrões. 
Tabela 1. Dados antropométricos das pacientes estudadas (média \pm DP e variação entre parênteses).

\section{$\mathbf{N}=\mathbf{5 2}$}

idade (anos) peso $(\mathrm{kg})$ IMC $\left(\mathrm{kg} / \mathrm{m}^{2}\right)$ cintura $(\mathrm{cm})$ $\mathrm{RCQ}$

$$
\begin{gathered}
37,5 \pm 9,4(19-54) \\
92,9 \pm 18,3(67-132) \\
36,4 \pm 6,4(20,7-49,4) \\
100,3 \pm 13,2(67-132) \\
0,84 \pm 0,10(0,65-1,04)
\end{gathered}
$$

Tabela 2. Estudo da área de gordura subcutânea, de gordura visceral e do somatório do volume das adrenais das pacientes estudadas (média \pm DP e variação entre parênteses).

$\mathrm{N}=\mathbf{5 2}$

Área de gordura subcutânea $\left(\mathrm{cm}^{2}\right)$

$436,5 \pm 163,6(74,2-746,8)$

Área de gordura visceral $\left(\mathrm{cm}^{2}\right)$

$158,7 \pm 63,4(37,0-328,2)$

Volume supra-renal

$\mathrm{E}+\mathrm{D}\left(\mathrm{cm}^{3}\right)$

$3,32 \pm 1,52(0,75-6,74)$

Estudando as diversas variáveis (Tabela 3), observa-se uma correlação positiva do peso com a medida da cintura $(r=0,817)$, com a $\mathrm{GV}(\mathrm{r}=0,396)$ e com a GSC $(r=0,813)$. Todas ao nível de significância de $\mathrm{p}<0,001$, exceto com a GV que apresentou um $p=0,002$. Da mesma forma, o IMC mostrou forte correlação positiva com a cintura $(\mathrm{r}=$ $0,879)$, com a GV $(\mathrm{r}=0,497)$ e com a GSC ( $\mathrm{r}=$ $0,795)$, também, com nível de significância de $\mathrm{p}<$ 0,001 . O IMC também se correlacionou positivamente com a RCQ, embora com uma significância menor $(r=0,262, p=0,030)$. Estes resultados não são surpreendentes, uma vez que se espera que o maior grau de corpulência tenha lincaridade com as medidas antropométricas, ou com as medidas diretas de gordura estudadas pela TC. A medida do peso não mostra relação com a $\mathrm{RCQ}$, o que também é coerente, já que o peso não indica maior ou menor deposição central de gordura. $O$ estranho é que o $\mathrm{IMC}$ tenha se correlacionado com a $\mathrm{RCQ}$, que revela mais adequadamente uma distribuição central, embora com uma significância menor

$O$ estudo das medidas de distribuição central da gordura (Tabela 3), como a medida da cintura abdominal e a RCQ, mostrou que a cintura se correlacionou positivamente com a $\mathrm{GV}(\mathrm{r}=0,584)$ e com a GSC $(r=0,710)$, com grau de significância $\mathrm{p}<0,001$. A RCQ apresentou correlação significante com a GV $(r=0,471, p<0,001)$, entretanto, não mostrou correlação com a GSC.

Aqui aparece a primeira evidência da proposição do estudo, quando a RCQ se mostrou positivamente correlacionada com a soma do volume das adrenais $(\mathrm{r}=0,272, \mathrm{p}=0,02)$ (Figura $\mathrm{I})$. Além disso, a área de $\mathrm{GV}$, medida por TC, que se correlacionou com a cintura $(\mathrm{r}=0,584)$, mostrou uma correlação direta com o volume das adrenais (Figura 2), embora no limite da significância $(\mathrm{r}=0,228, \mathrm{p}=0,05)$.

Para avaliar se havia diferença entre o volume das adrenais e a GV, as pacientes foram divididas em 2 grupos, área de $\mathrm{GV}<\mathrm{e} \geq 120 \mathrm{~cm}^{2}$, utilizado o teste de Levene para testar a igualdade das variáveis. A correlação entre o volume das adrenais e a GV se mostrou positiva no limite da significância $(p=0,05)$. Portanto, no grupo de pacientes com GV $\geq 120 \mathrm{~cm}^{2}$, houve uma correlação positiva com o somatório do volume das adrenais.

Tabela 3. Correlações entre os dados antropométricos e tomográficos das pacientes estudadas.

\begin{tabular}{llllllll}
\hline & $\begin{array}{l}\text { Peso } \\
(\mathbf{k g})\end{array}$ & $\begin{array}{l}\text { IMC } \\
\left(\mathbf{k g} / \mathbf{m}^{2}\right)\end{array}$ & $\begin{array}{l}\text { Cintura } \\
\mathbf{( c m )}\end{array}$ & RCQ & $\begin{array}{l}\text { GV } \\
\left(\mathbf{c m}^{2}\right)\end{array}$ & $\begin{array}{l}\text { GSC } \\
\left(\mathbf{c m}^{2}\right)\end{array}$ & $\begin{array}{l}\text { VSRD+E } \\
\left(\mathbf{c m}^{3}\right)\end{array}$ \\
Peso & - & $0,930^{*}$ & $0,817^{*}$ & 0,168 & $0,396^{* * *}$ & $0,813^{*}$ & $-0,079$ \\
IMC & $0,930^{*}$ & - & $0,879^{*}$ & $0,262^{* *}$ & $0,497^{*}$ & $0,795^{*}$ & $-0,070$ \\
Cintura & $0,817^{*}$ & $0,879^{*}$ & - & $0,627^{*}$ & $0,584^{*}$ & $0,710^{*}$ & 0,060 \\
RCQ & 0,168 & $0,262^{* *}$ & $0,627^{*}$ & - & $0,471^{*}$ & 0,106 & $0,272^{* *}$ \\
AGV & $0,396^{* * *}$ & $0,497^{*}$ & $0,584^{*}$ & $0,471^{*}$ & - & $0,297^{* *}$ & $0,228 \#$ \\
AGSC & $0,813^{*}$ & $0,795^{*}$ & $0,710^{*}$ & 0,106 & $0,297^{* *}$ & - & $-0,005$ \\
VSRD+E & $-0,079$ & $-0,070$ & 0,060 & $0,272^{* *}$ & $0.228 \#$ & $-0,005$ & - \\
\hline
\end{tabular}

$* \mathrm{p}<0,001$

${ }^{* *} p<0,05$ e $>0,01$

${ }^{* * *} \mathrm{p}<0,005$

$\# \mathrm{p}=0,05$.

IMC = Índice de massa corporal

$R C Q=$ relação cintura/quadril

$A G V=$ área de gordura visceral

$A G S C$ = área de gordura subcutânea

$\mathrm{VSRD}+\mathrm{E}=$ somatório do volume das supra-renais. 


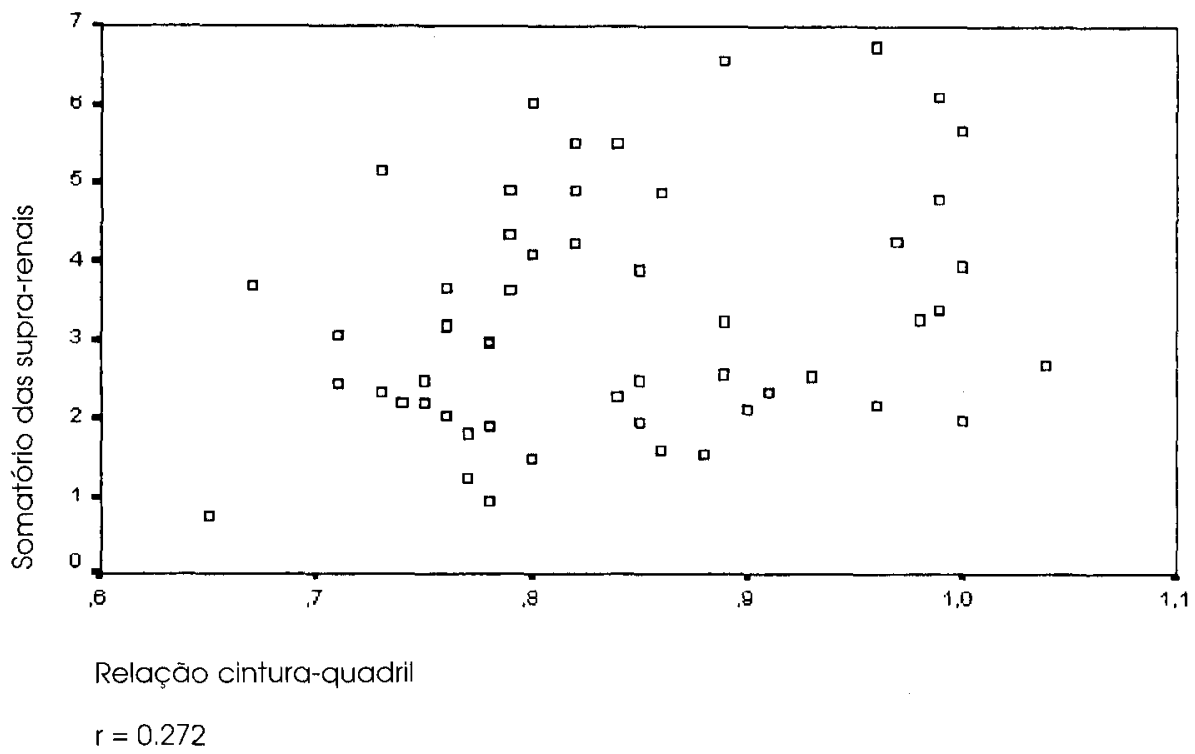

Figura 1. Correlação entre a relação cintura/quadril $(R C Q)$ e o somatório do volume das adrenais mensurado por tomografia computadorizada.

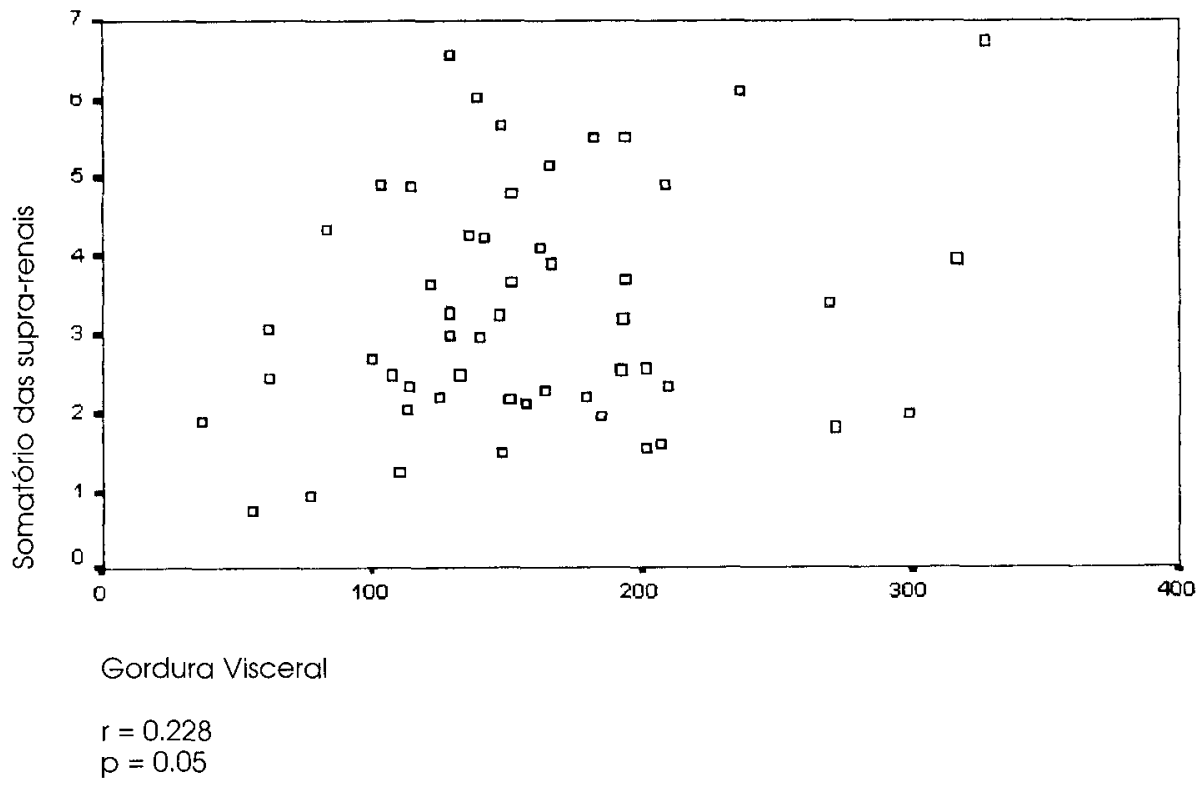

Figura 2. Correlação entre a área de gordura visceral e o somatório do volume das adrenais mensurados por tomografia computadorizada.

\section{DISCUSSÃO}

O estudo de avaliação da deposição central de gordura tem como padrão-ouro as medidas realizadas por TC $(38,39)$, porque este método avalia diretamente a área visceral $\mathrm{e} / \mathrm{ou}$ subcutânea. Quando estas medidas são correlacionadas com parâmetros de mais fácil aferição, como as medidas da cintura e a RCQ, observa- se em geral um bom índice de precisão destas medidas indiretas $(16,19-25)$. Pouliot e col. (25) demonstraram que a simples medida da cintura foi um melhor indicador de acúmulo de GV do que a RCQ. Os resultados obtidos no presente estudo, embora em geral concordantes com o destes autores e de outros, apresenta algumas peculiaridades. A medida da cintura avaliou positivamente tanto a GV como a GSC, mas o 
coeficiente foi maior com a GSC do que com a GV. O que chama mais atenção é que neste estudo a RCQ se correlacionou com a GV, mas não com a GSC, sugerindo que a RCQ prediz melhor a gordura intraabdominal do que a gordura subcutânea. Entretanto, quando analisamos a GV e sua correlação com as medidas antropométricas, observamos que, de fato, há uma maior correlação com a cintura $(\mathbf{r}=0,584)$ do que com a $\mathrm{RCQ}(\mathrm{r}=0,471)$, o que é concordante com outros autores (21-23,38).

O maior propósito deste estudo, em verdade, foi o de procurar uma correlaçào anatômica para as possíveis modificações funcionais do eixo HHA na obesidade central. Com efeito, as evidências apontam para uma hiperatividade do eixo HHA em pacientes com obesidade central $(26,29,30)$. Marin e col. (26) observaram maiores níveis de cortisol urinário quanto maior a RCQ. Pacientes submetidos a testes de stress psicológicos, como testes matemáti$\cos$ e de quebra-cabeças, revelam uma resposta do cortisol plasmático que se correlaciona com o diâmetro sagital, uma medida precisa de deposição abdominal de gordura (26). Além disso, uma maior deposição central de gordura está associada a uma menor supressibilidade do cortisol à dexametasona (31). Utilizando injeções de hormônio adreno-corticotrófico (ACTH) ou de hormônio liberador de adreno-corticotrofina (CRH), Pasquali e col. (29) demonstraram que mulheres com distribuição abdominal de gordura apresentavam resposta do cortisol e do ACTH maior que as normais e ainda maior que aquelas com deposição periférica. O mesmo grupo (30) demonstrou que injetando-se $\mathrm{CRH}$ e arginina vasopressina (AVP), um potencializador da ação do CRH, a resposta do ACTH e do cortisol foi significativamente maior no grupo com acúmulo de gordura visceral ( $\mathrm{RCQ}=0,88 \mathrm{e} \mathrm{GV}=$ $186,5 \mathrm{~cm}^{2}$ ), e que durante os testes de stress (teste matemático e de quebra-cabeças) o grupo com maior acúmulo visceral apresentou uma frequêencia cardíaca maior durante estes testes, sugerindo que estas mulheres apresentam uma resposta simpática hiperativa ao stress agudo e que isto parece interrelacionar-se com o eixo HHA. Sugere-se que a obesidade visceral depende de vários fatores, incluindo a má adaptação ao stress crônico (1,39-42). Estudos em animais claramente demonstram que similares alterações neuroendócrinas ocorrem durante uma reação a fatores estressantes (43). Em situações de não stress, tanto o CRF quanto a AVP, são secretados no sistema porta-hipofisário de maneira pulsátil, com boa concordância entre os pulsos (34).
Mas, durante exposição ao stress, a amplitude dos níveis de AVP aumenta (34). Nestes animais, tanto as concentrações de AVP quanto CRH aumentam quando injeta-se norepinefrina intracerebroventricular (44). A ativação do sistema simpático representa, assim, o principal fator de regulação endócrina de adaptação ao stress (45), e parece estar significativamente correlacionada com a ativação do HHA (46). A hiperatividade do sistema simpático e do HHA após stress agudo tem sido observado, por exemplo, em pessoas com alta reatividade cardíaca ao stress (46). No trabalho de Pasquali, os achados de maior freqüência cardíaca e maiores níveis de cortisol no grupo com acúmulo de GV confirmam esta hipótese. Portanto, uma das hipóteses é de que mulheres com maior adiposidade visceral representem uma classe de indivíduos que tem uma hiperatividade simpática relacionada ao stress e esta pode estar associada com uma hiperatividade do HHA. Mais recentemente, e utilizando uma tecnologia mais sofisticada, através de medidas seqüenciais do cortisol salivar ao longo de um dia, foi possível observar uma menor variabilidade do cortisol em resposta a percepção ao stress, associada a uma defeituosa supressão com dexametasona em relação a obesidade abdominal, indicando uma anormalidade do eixo HHA neste grupo (47).

Em função das anormalidades descritas acima, poder-se-ia esperar que um eixo HHA cronicamente estimulado na obesidade central culminaria com alguma repercussão no tamanho da glândula alvo, a adrenal. Com o mesmo tipo de raciocínio, se demonstrou que pacientes com depressão endógena, uma sabida situação de anormalidade do eixo HHA $(33,34)$, se acompanha de aumento do volume das adrenais. Ainda mais, a melhora dos sintomas depressivos é acompanhada por uma diminuição do volume das adrenais para níveis semelhantes aos do grupo controle (35).

É possível afirmar que o presente estudo logrou demonstrar o que se suspeitava. Por exemplo, a RCQ avaliou mais adequadamente a gordura visceral que a gordura subcutânea, com a qual não houve correlação. Foi exatamente esta medida que mais sensivelmente se correlacionou com o volume das adrenais (Figura 1) $(\mathrm{r}=0,272, \mathrm{p}=0,02)$. Era de se esperar que, também, houvesse uma relação direta entre a GV e o volume das adrenais, e de fato esta correlação existiu $(r=0,228, p=$ 0,05 ) (Figura 2), porém no limite da significância. É possivel que a escolha das pacientes tenha interferido nestes resultados, diminuindo o coeficiente de relação, já que, sabidamente, a obesidade central está mais comumente associada a menores graus de obesidade, 
sendo o grupo aqui estudado de IMC médio elevado. Além disso, na tentativa de estudar uma larga variação de corpulência, as voluntárias normais foram de número reduzido (apenas 5) e nenhuma paciente estava numa faixa intermediária de IMC (entre 25 e $30 \mathrm{~kg} / \mathrm{m}^{2}$ ).

É importante observar que o volume das adrenais não se correlacionou com qualquer outro parâmetro estudado, como o peso e o IMC, sugerindo, portanto, que não é a obesidade, mas sim a centralização de gordura, que guarda esta relação. Ainda mais, diferentemente da relação entre a GV $c$ somatório das adrenais, não houve qualquer relação do volume adrenal com a GSC, o que leva a outra evidência de que o HHA está envolvido não na deposição subcutânea de gordura, mas sim na visceral.

$\mathrm{Na}$ tentativa de reforçar os achados, as pacientes foram divididas entre aquelas com GV $<\mathrm{e} \geq 120 \mathrm{~cm}^{2}$, e comparados os volumes das adrenais. $\mathrm{O}$ resultado mostrou, que o grupo com GV $\geq 120 \mathrm{~cm}^{2}$, apresentava um volume adrenal maior $\left(3,56 \pm 1,5 \mathrm{lcm}^{3}\right)$ $(\mathrm{p}=0,05)$ que o grupo com GV $<120 \mathrm{~cm}^{2}(2,62 \pm$ $\left.1,37 \mathrm{~cm}^{3}\right)$, confirmando que maiores áreas de GV estão associadas a um aumento das adrenais. $O$ ponto de corte dos grupos, em torno de $120 \mathrm{~cm}^{2}$, está de acordo com estudos que sugerem que as alterações metabólicas da obesidade visceral se incrementam a partir de uma área em torno de $130 \mathrm{~cm}^{2}$, valor próximo ao ponto de corte utilizado neste estudo (17). Além disso, realizou-se vários pontos de corte até alcançar este valor, que mostrou significância com o volume das adrenais.

Além da contribuição da hiperatividade do eixo HHA, estimulando as glândulas adrenais, é possível que outros fatores também levem a este aumento do volume glandular. O maior aporte de ácidos graxos livres advindos do tecido adiposo visceral para o figado contribuem para um clearence diminuído de insulina pelo figado e, consequentemente, leva a hiperinsulinemia (4,48-50). As alterações endócrinas, o hipercortisolismo, o aumento de testosterona livre (em mulheres) e a diminuição de SHBG, todos estes fatores contribuem para a resistência insulínica e hiperinsulinemia (3,51-55). Em trabalho recente, Reinckle e col. (56) demonstraram que células adrenais da linhagem NCI-h295, quando incubadas com concentrações diferentes de insulina, produziram uma atividade mitótica acentuada relacionada com o tempo e com as quantidades crescentes de insulina. Isto sugere que talvez a própria hiperinsulinemia, encontrada em pacientes com maior acúmulo de gordura visceral, e a hiperatividade do HHA, estimulariam o aumento do volume das adrenais o que culminaria num ciclo vicioso: hiperatividade HHA aumento das adrenais - aumento da gordura visceral - resistência à insulina - hiperinsulinemia - maior aumento das adrenais (Figura 3 ).

Por outro lado, embora este seja um modelo factível para explicar esta relação $\mathrm{GV} X$ volume das adrenais, há sugestões de que o cortisol advém de uma maior conversão no tecido adiposo omental $(57,58)$. Um dos estudos demonstra, através da incubação do tecido adiposo subcutâneo e omental, que a atividade da enzima oxo-redutase é maior no tecido omental e que esta atividade foi maior quando se adicionou cortisol e insulina. A oxo-redutase transforma a cortisona em cortisol, favorecendo portanto uma inỵersão do metabolismo. Níveis maiores de cortisol gerado nestas células poderia promover, de maneira autócrina nas

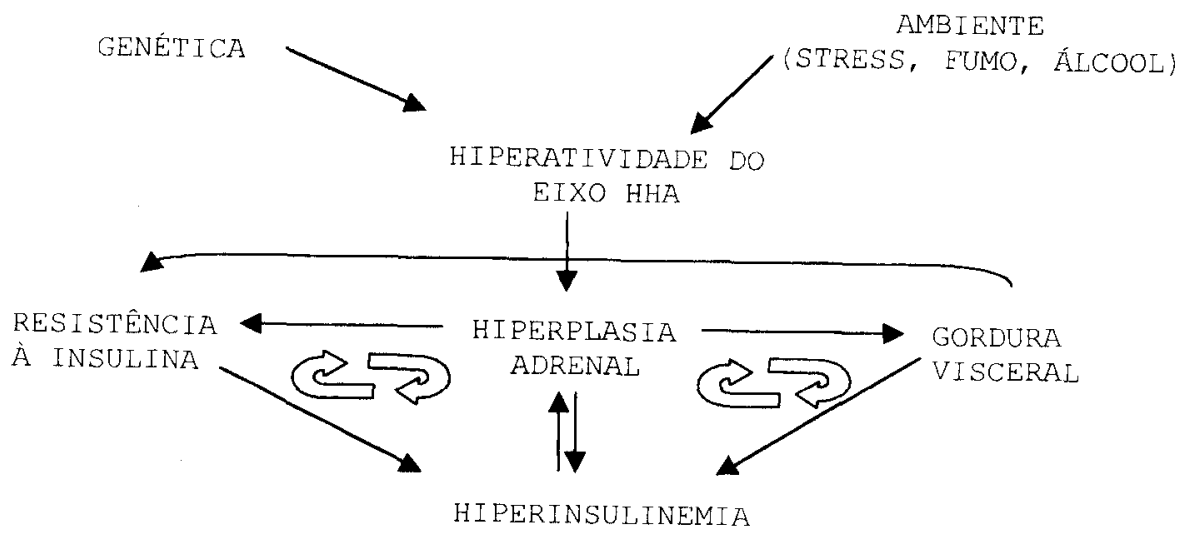

Figura 3. Modelo de interação entre o eixo hipotálamo-hipofisário-adrenal (HHA) e a Síndrome Metabólica. 
células estromais e de maneira parácrina nos adipócitos adjacentes, o acúmulo de gordura à nível visceral ("Cushing omental").

\section{CONCLUSŌES}

Nosso estudo sugere que a medida da cintura é um ótimo preditor de GV, mas não diferencia da GSC. Já a $\mathrm{RCQ}$ só se correlacionou com a GV, que é a medida antropométrica que justamente se correlaciona com o volume das adrenais. Não há qualquer correlação entre a GSC e o somatório do volume das adrenais. Portanto, o teor de GV está diretamente relacionado a um maior volume das adrenais, sugerindo que exista uma associação fisiopatológica. Uma conclusão importante é que não é a obesidade per si, mas a centralização ou, mais especificamente, a GV que mantém algum grau de associação com o eixo HHA.

\section{REFERÊNCIAS}

1. Björntörp P. Metabolic implications of body fat distribution. Diabetes Care 1991:14:1132-43.

2. Vague J. The degree of masculine differentiation of obesities: a factor determining predisposition to diabetes, atherosclerosis, gout and uric-calculus disease. Am J Clin Nutr 1956:4:20-34.

3. Björntörp P. Endocrine abnormalities in obesity. Diabetes Rev 1997;5:52-68.

4. Kissebah AH, Petris AN. Biology of regional body fats distribution: relationship to non-insulin-dependent diabetes mellitus. Diabetes Metab Rev 1989;5:83-100.

5. Després JP, Moorjani S, Ferland M. Adipose tissue distribution and plasma lipoprotein levels in obese women: importance of intra-abdominal fat. Arteriosclerosis 1989:9:203-10.

6. Haffner SM, Stern MP. Hazuda HP, et al. Do upper-body and centralized obesity measure different aspects of regional body fat distribution? Relationship to non-insulindependent diabetes mellitus, lipids and lipoproteins. Diabetes 1987;36:43-51.

7. Kalhoff RK, Hartz AH, Rupley D, et al. Relationship of body fat distribution to blood pressure carbohydrate tolerance, and plasma lipids in healthy obese women. J Lab Clin Med 1983; 102:621-7.

8. Krotkiewskl M, Björntörp P. Sjöstrom L, et al. Impact of obesity on metabolism in men and women. Importance of regional adipose tissue distribution. J Clin Invest $1983 ; 72: 1150-62$

9. Lapidus L, Bengtsson C, Larsson B, et al. Distribution of adipose tissue and risk of cardiovascular disease and death: a 12-year follow-up of participants in the population study of women and Gothenburg, Sweden. Br Med J 1984;289:1257-61.

10. Larsson B, Svardsudd K, Wilhelmsen L, et al. Abdominal adipose tissue distribution, obesity and risk of cardiovascular disease and death: 13-year follow-up of participants in the study of men born in 1923. Br Med J 1984:288: 1401-4.

11. Peiris AN, Hennes MI, Evans DJ, et al. Relationship of anthropometric measurements of body fat distribution to metabolic profile in premenopausal women. Acta Med Scand Suppl 1988;723:179-88.

12. Peiris AN, Sothmann MS, Hoffmann RG, et al. Adiposity, fat distribution and cardiovascular risk. Ann Intern Med 1989: 1 10:867-72.

13. Björntörp P. "Portal" adipose tissue as a generator of risk factors for cardiovascular disease and diabetes. Arteriosclerosis 1990; 10:493-6.

14. Després JP, Nadeau A, Tremblay A, et al. Role of deep abdominal fat in the association between regional adipose tissue distribution and glucose tolerance in obese women. Diabetes 1989;38:304-9.

15. Després JP, Moorjani S, Lupien PJ, et al. Regional distribution of body fat, plasma lipoproteins and cardiovascular disease. Arteriosclerosis 1990: 10:497-511.

16. Fujioka S, Matsuzawa $Y$, Tahuna K, et al. Contribution of intra-abdominal fat accumulation to the impairment of glucose and lipid metabolism in human obesity. Metabolism 1987:36:54-9

17. Lemieux S. Prud'Homme D. Nadeau A, et al. Seven year changes in body fat and visceral adipose tissue in women. Associations with indexes of plasma glucose-insulin homeostasis. Diabetes Care 1996;19:98391.

18. Pouliot MC, Després JP, Nadeau A, et al. Visceral obesity in men: associations with glucose tolerance, plasma insulin, and lipoprotein levels. Diabetes 1992;41:826-34.

19. Ashwell M, Cole TJ, Dixon AK, et al. Obesity: new insight into the anthropometric classification of fat distribution shown by computed tomography. Br Med J 1985:290: 1692-4.

20. Ferland M, Després JP, Tremblay A, et al. Assessment of adipose tissue distribution by computed axial tomography in obese women: association with body density and anthropometric measurements. Br J Nutr $1989 ; 61: 139-48$

21. Seidel JC, Oosterlee A, Duerenberg P, et al, Abdominal fat depots measured with computed tomography: effects of degree of obesity, sex and age. Eur J Clin Nutr 1988:42:805-15

22. Borkan GA, Hults DE, Gerzof SG, et al. Relationships between computed tomography tissue areas, thickness and total body composition. Ann Hum Biol 1983: 10:537-46

23. Després JP, Prud'Homme D. Tremblay MC, et al. Estimation of deep abdominal adipose tissue accumulation from simple anthropometric measurements to men. Am J Clin Nutr 1991:54:471-7.

24. Kissebah AH. Central obesity: measurement and metabolic effects. Diabetes Rev 1997:5:8-21.

Arq Bras Endocrinol Metab vol $44 n^{\circ} 1$ Fevereiro 2000 
25. Pouliot M, Després J, Lemieux S, et al. Waist circumference and abdominal sagital diameter: best simple anthropometric indexes of abdominal visceral adipose tissue accumulation and related cardiovascular risk in men and women. Am J Cardiol 1994;73:460-8.

26. Marin P, Darin N, Amemiya T, et al. Cortisol secretion in relation to body fat distribution in obese premenopausal women. Metabolism 1992;41:882-6.

27. Strain GW. Zumoff B. Strain JJ, et al. Cortisol production in obesity. Metabolism 1980;29:980-5.

28. Moyer AE, Rodin J, Grilo CH, et al. Stress-induced cortisol response and fat distribution in women. Obes Res $1994 ; 2: 255-62$.

29. Pasquali R, Cantobelli S, Casimiri F, et al. The hypothalamic-pituitary-adrenal axis in obese women with different patterns of body fat distribution. J Clin Endocr Metab 1993:77:341-6.

30. Pasquali $R$, Anconetani $B$, Chattat $R$, et al. Hypothalamic-pituitary-adrenal axis activity and its relationship to the autonomic nervous system in women with visceral and subcutaneous obesity: effects of the corticotrophin-releasing factor/arginine-vasopressin test and of stress. Metabolism 1996:45:351-6.

31. Ljung T, Anderson B, Björntörp P, et al. Inhibition of cortisol secretion by dexamethasone in relation to body fat distribution: a dose-response-study. Obes Res 1996:4:277-82.

32. Björntörp $P$. The regulation of adipose tissue distribution in humans. Int J Obes 1996:20:291-302.

33. Linkowsk| P, Mendlewicz J, Leclercq R, et al. The 24hour profile of adrenocorticotropin and cortisol in major depressive illness. J Clin Endocrinol Metab 1985:61:429-38.

34. Tsigos C. Chrousos GP. Physiology of the hypothalamicpituitary-adrenal axis in health and disregulation in psychiatric and autoimmune disorders. Endocrinol Metab Clin North Am 1994:23:451-66.

35. Rubbin RT, Phillips JJ, Sadow TF, et al. Adrenal gland volume in major depression. Increase during the depressive episode and decrease with successful treatment. Arch Gen Psych 1995;52:213-8.

36. Amsterdam JD, Marinelli DL, Arger P, et al. Assessment of adrenal gland volume by computed tomography in depressed patients and healthy volunteers: a pilot study. Psych Res 1987;21:189-97.

37. Sjöstrom L, Kvist $H$, Cederland A, et al. Determination of total adipose tissue and body fat in women by computed tomography, $40 \mathrm{~K}$ and tritium. Am J Physiol 1986;250:E736-E745

38. Ferland M, Després JP. Tremblay A, et al Assessment of adipose tissue distribution by computed axial tomography in obese women: association with body density in and anthropometric measurements. $\mathbf{B r} \mathbf{J}$ Nutr 1989;61:139-48.

39. Lapidus L, Bengstsson C, Hällström T, et al. Obesity, adipose tissue distribution and health in women-results from a population study in Gothenburg, Sweden. Appetite $1989 ; 12: 25-35$.

40. Larsson B, Seidell J, Svärdsudd K, et al. Obesity, adipose tissue distribution and health in men the study of men born 1913. Appetite 1989;13:37-44.

41. Rosmond R, Lapidus L, Marin P, et al. Mental distress, obesity and body fat distribution in middle-aged men. Obes Res 1996:4:245-52.

42. Wing RR, Mathews KA, Kuller LH, et al. Waist to hip ratio in middle-aged women. An association with behavioral and psychosocial factors and with changes in cardiovascular risk factors. Arterioscler Thromb 1991;11:1250-7.

43. Seeman TE, Robbins RJ. Aging and hypothalamic-pituitary-adrenal response to challenge in humans. Endocr Rev 1994; 15:233-60

44. Lin JP, Clarke IJ, Funder JW, et al. Studies of the secretion of corticotrophin releasing factor and arginine vasopressin into the hypophysial-portal circulation of conscious sheep. J Clin Invest 1994;93: 1439-50.

45. Chrousos GP, Gold PW. The concept of stress and stress system disorders. Overview of physical and behavioral homeostasis. JAMA 1992;267:1244-52.

46. Cacioppo JT. Social neuroscience: autonomic, neuroendocrine and immune responses to stress. Psychophysiology 1993:31:113-28.

47. Rosmond R, Dallman MF, Björntörp P. Stress-related cortisol secretion in men: relationships with abdominal obesity and endocrine, metabolic and hemodynamic abnormalities. J Clin Endocrinol Metab 1988:83: 1853-9.

48. Björntörp P. Abdominal obesity and the development of NIDDM. Diabetes Metab Rev 1988;4:615-22.

49. Després JP, Lemieux S, Lamarche B, et al. The insulin resistance-dyslipidemic syndrome: contribution of visceral obesity and therapeutic implications. Int J Obes 1995: 19:576-\$86.

50. Després JP. Abdominal obesity as important component of insulin-resistance syndrome. Nutrition 1993;9:452-9.

51. Després JP. Lipoprotein metabolism in visceral obesity. Int J Obes 1991;15:45-52

52. Guillaume-Gentil C, Assimacopoulos-Heannet F, Jeaurenaud B. Involvement of non-esterified fatty acid oxidation in glucocorticoid-induced peripheral insulin resistance in vivo in rat. Diabetologia 1993:36:899-906

53. Haffner SM, Valdez RA, Morales PA, et al. Decreased sex hormone-binding globulin predicts non-insulin-dependent diabetes mellitus in women but not in men. J Clin Endocrinol Metab 1993:77:56-60

54. Jayo J, Shively C, Kaplan J, et al. Effects of exercise and stress on body fat distribution in male Cynamolgus monkeys. Int J Obes 1993; 17:597-604.

55. McMahon M, Gerich J, Rizza R. Effects of glucocorticoids on carbohydrate metabolism. Diabetes Metab Rev 1988;4:17-30. 
56. Reinckle $M$, Fabnacht $S$, Väth $S$, et al. Adrenal incidentalomas: a manifestation of the metabolic syndrome. Endocr Res 1996:22:757-61.

57. Andrew R, Phillips A, Walker BR. Obesity and gender influence cortisol secretion and metabolism in man. $J$ Clin Endocrinol Metab 1998:83:1806-9.

58. Bujalska IJ, Kurnar S, Stewart PM. Does central obesity reflect "Cushing's disease of the omentum"? Lancet 1997:349:1210-3.

Endereço para correspondência:

Amélio F. de Godoy Matos

R. Visconde Silva 52, sl. 704

22271-090 Rio de Janeiro, RJ. 